$\begin{array}{cl}\text { PRAMANA } & \text { (C) Indian Academy of Sciences } \\ \begin{array}{c}\text { journal of } \\ \text { physics }\end{array} & \begin{array}{l}\text { Vol. } 69, \text { No. } 6 \\ \text { December } 2007 \\ \text { pp. } 1171-1175\end{array}\end{array}$

\title{
Study on low-energy positron polarimetry
}

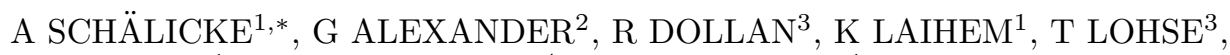 \\ S RIEMANN $^{1}$, P STAROVOITOV $^{4}$ and A USHAKOV ${ }^{1}$ \\ ${ }^{1}$ DESY, Platanenallee 6, 15738 Zeuthen, Germany \\ ${ }^{2}$ Physics Department, Tel Aviv University, Tel Aviv 69978, Israel \\ ${ }^{3}$ Physics Department, Humboldt University, Newtonstr. 15, 12489 Berlin, Germany \\ ${ }^{4}$ National Center for Particle and High Energy Physics, Bogdanovitch Street 153, \\ 220040 Minsk, Belarus \\ *E-mail: andreas.schaelicke@desy.de
}

\begin{abstract}
A polarised positron source has been proposed for the design of the international linear collider (ILC). In order to optimise the positron beam, a measurement of its degree of polarisation close to the positron creation point is desired. In this contribution, methods for determining the positron polarisation at low energies are reviewed. A newly developed polarisation extension to GEANT4 will provide the basis for further polarimeter investigations.
\end{abstract}

Keywords. Positron source; polarimetry; Monte Carlo simulation; GEANT4.

PACS Nos 24.10.Lx; 13.88.+e; 29.27.Hj

\section{Introduction}

At an ILC the possibility to have both beams - electrons and positrons - polarised will substantially broaden its physics potential [1]. While polarised electron sources exist already, the design and optimisation of a polarised positron source is the subject of current R\&D projects [2,3].

Polarised positrons are created from circularly polarised photons hitting a thin target. The spin is transferred to the pair-produced electrons and positrons. The positrons are captured right behind the target with dedicated capture optics, i.e. an adiabatic matching device. In the nominal design of the ILC the positron beam pulse consists of 2820 bunches containing $2 \cdot 10^{10}$ positrons each; pulse repetition rate is $5 \mathrm{~Hz}$.

In order to optimise the positron beam, i.e. to ensure a high intensity as well as high degree of polarisation, a measurement of the polarisation close to the positron creation point is desired. Depending on the position of the low-energy polarimeter, the positron energy $E_{e^{+}} \approx 30-40 \mathrm{MeV}$ behind the capture section, $E_{e^{+}} \approx 100-300$ $\mathrm{MeV}$ behind the pre-accelerator and $E_{e^{+}} \approx 5 \mathrm{GeV}$ near the damping ring. The 


\section{A Schälicke et al}

typical transverse beam size is $\sim 1 \mathrm{~cm}$. In this paper, different options for a positron polarimeter at the positron source will be evaluated.

\section{Evaluation of methods}

Possibilities to measure the polarisation of positrons (or electrons) are:

- Laser Compton polarimeter [4]:

A laser Compton polarimeter is the recommended option for a polarimeter close to the interaction point. Laser photons hit the positron (or electron) beam and are backscattered; the angular distribution depends on the polarisation of the positrons. For low-energy positron beams, the asymmetry in the angular distribution of the backscattered photons is very small. In addition, due to the large emittance of the positron beam before the damping ring, the signal rate is too low.

- Compton transmission polarimeter [5]:

For photons of a few $\mathrm{MeV}$, Compton transmission polarimetry is a wellestablished method. It relies on the fact that the transmission of a photon beam through iron depends on the polarisation of the beam photons as well as on the magnetisation of the iron target. Reversing the polarity of the magnetic field in iron results in an asymmetry of the transmission signal at the per cent level. This method can also be applied to positrons (or electrons), after their reconversion to photons in a bremsstrahlung target. It has been employed both in the E166 experiment at SLAC $\left(E_{e^{+}} \approx 3-8 \mathrm{MeV}\right)[2]$ and in the ATF experiment at $\mathrm{KEK}\left(E_{e^{+}} \approx 30-40 \mathrm{MeV}\right)[3]$. However, at energies higher than a few tens of $\mathrm{MeV}$ this method becomes inefficient, since with rising energy the cross-section for pair production becomes more and more dominant over the Compton process. Furthermore, the method is destructive, i.e. at least one complete bunch of a pulse will be dumped for the polarisation measurement.

- Mott scattering [6]:

A polarimeter based on Mott scattering works with transversely polarised beams. In the relevant energy range the cross-section of elastic positron/electron-nucleus scattering decreases more rapidly than that of Bhabha and Møller scatterings. The corresponding event rate is too low at the damping ring.

- Spin-dependent synchrotron radiation [7]:

Polarisation measurements based on the fact that synchrotron radiation depends on the orientation of the electron/positron spin in a magnetic field have been successfully demonstrated at the VEPP-4 storage ring. Asymmetries of the synchrotron radiation created in a three pole magnetic 'snake' could also be used in the ILC damping ring to monitor the transverse polarisation of the positrons. This method is non-destructive and non-intrusive but the expected asymmetries are very small, i.e., $\leq 10^{-3}$. The short storage period in the damping ring makes it difficult to reduce the statistical and systematic uncertainties enough to measure this effect. 


\section{Low-energy positron polarimetry}

- Bhabha/Møller polarimeter [8,9]:

This method is discussed in the next section for the example of Bhabha scattering.

\section{Bhabha polarimetry at low energies}

The cross-section for Bhabha scattering may be written in the center-of-mass system as

$$
\begin{aligned}
\frac{\mathrm{d} \sigma}{\mathrm{d} \Omega} \sim & \frac{(1+\cos \theta)^{2}}{16 \gamma^{2} \sin ^{4} \theta} \\
& \times\left\{\left(9+6 \cos ^{2} \theta+\cos ^{4} \theta\right)-P_{e^{+}} P_{e^{-}}\left(7-6 \cos ^{2} \theta-\cos ^{4} \theta\right)\right\}
\end{aligned}
$$

If both incoming particles are longitudinally polarised, an asymmetry up to $7 / 9 P_{e^{+}} P_{e^{-}}$can be obtained at scattering angles $\theta \rightarrow \pi / 2$ (see left part of figure 1 ). This is used in polarimeters by scattering positrons, with a polarisation $P_{e^{+}}$, on the shell electrons of $\mathrm{Fe}$ atoms in a thin magnetised iron foil, corresponding to an effective target polarisation $P_{e^{-}}$of $\approx 8 \%$. The asymmetry is measured by either reversing the magnetic field of the target or flipping the spin of the beam particles. This method can be exploited in a relatively simple set-up. However, operation at high beam currents is problematic, since the target is heated up and may become depolarised. In addition, the electromagnetic field of an intense beam may destroy the target polarisation. Møller polarimeters are widely used, e.g. in SLAC fixed target experiments and at the VEPP-3 storage ring (gas target) [8].

At low positron energies, Bhabha polarimetry seems to work with an acceptable signal-to-background ratio. According to the results of earlier studies [9], it is recommended to place the polarimeter behind the positron pre-accelerator $\left(E_{e+} \approx 200\right.$ $\mathrm{MeV}$ ) at the point where the positron beam is separated from electron and photon beams. The main source of background is bremsstrahlung, and Bhabha events have to be separated from Mott scattering and annihilation in flight processes. Analysing only final-state electrons allows for an efficient suppression of bremsstrahlung background (see also the right part of figure 1).

The iron target could be a technical challenge. The critical temperature $T_{\mathrm{c}}=$ $1043 \mathrm{~K}$ and will be easily exceeded if an intense beam with a small beam size hits the target. The working temperature of the target has to be stable within a certain limit to guarantee reliable measurements of the asymmetry. With the large size of the low-energy positron beam, the temperature at the target should be within reasonable limits, and depolarisation by the electromagnetic field of the beam is negligible. The temperature evolution in a $30 \mu \mathrm{m}$ target has been calculated assuming a beam with $\sigma=1 \mathrm{~cm}, N_{e^{+}}=2 \cdot 10^{10}$ particles per bunch and an energy of $\approx 200 \mathrm{MeV}$. A heat-up of $\sim 10 \mathrm{~K}$ per pulse is obtained. Assuming cooling by radiation the peak temperature in equilibrium is $\sim 500 \mathrm{~K}$ using all bunches of the pulse. This temperature rise will result only in a slight reduction of the polarisation of the electrons in the iron foil to $\sim 93 \%$, which seems acceptable. The variation of the target polarisation within a pulse is well below $1 \%$, which is sufficient for the desired accuracy of the polarisation measurement. 

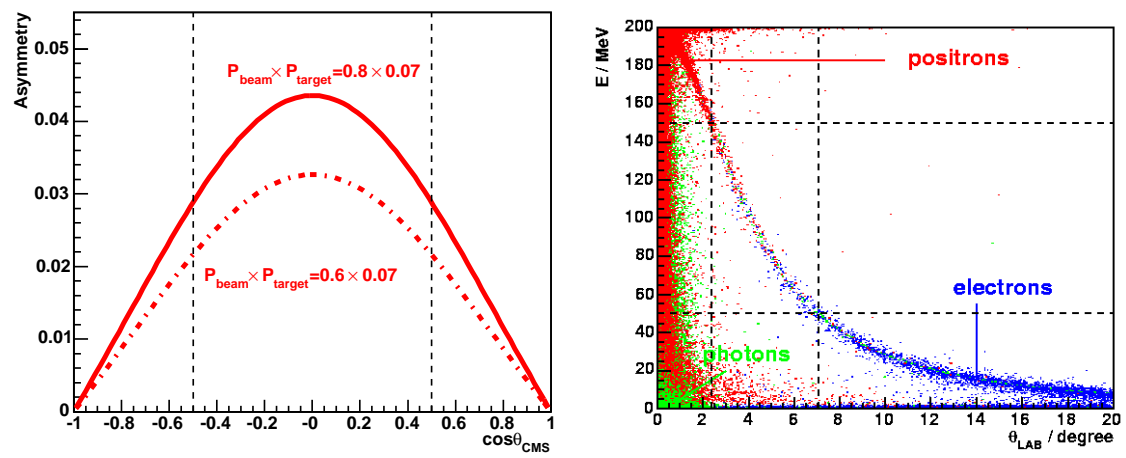

Figure 1. Left: Angular dependence of the asymmetry in Bhabha-polarimetry in the CMS frame for different beam (positron) and target (iron electron) polarisations. Right: Phase-space distribution of electrons, positrons and photons after passing $30 \mu \mathrm{m}$ iron foil. Positron beam energy $E_{e^{+}}=200 \mathrm{MeV}$. Dashed lines indicate the region of highest asymmetry in both plots.

\section{GEANT4 with polarisation}

The development of polarimeters for electrons or positrons requires reasonable simulations and modelling to evaluate the analysing power. For a complete simulation of a low-energy polarimeter set-up all processes relevant for tracking polarised particles through matter, i.e. spin dependence of Compton scattering, Bhabha/Møller scattering, annihilation into photons as well as the polarisation transfer via bremsstrahlung and pair production, are needed. Therefore, the GEANT4 package, 'a toolkit for the simulation of the passage of particles through matter' [10], is being extended by an independent polarisation library that will provide these polarised QED processes [11]. If needed polarisation can be assigned to each logical volume. Currently, this extension package is being checked against data from the E166 experiment. The aim is to include the library for polarised processes and a corresponding user code in the upcoming December release of GEANT4. An important application of the GEANT4 extension with polarisation is the design and optimisation of the positron production target, and a realistic simulation of a low-energy polarimeter.

\section{Acknowledgement}

This work is supported by the Commission of the European Communities under the 6th Framework Programme 'Structuring the European Research Area', contract number RIDS-011899, and by the ISF - Israel Science Foundation.

\section{References}

[1] G A Moortgat-Pick et al, arXiv:hep-ph/0507011 


\section{Low-energy positron polarimetry}

[2] K Laihem et al, These proceedings G Alexander et al, SLAC-TN-04-018

[3] T Omori et al, Phys. Rev. Lett. 96, 114801 (2006); arXiv:hep-ex/0508026

[4] V Gharibyan, N Meyners and P Schüler, LC-DET-2001-047 SLD Collaboration: M Woods, arXiv:hep-ex/9611005 M Beckmann et al, Nucl. Instrum. Methods A479, 334 (2002)

[5] H Schopper, Nucl. Instrum. Methods 3, 1958 (1960)

[6] T J Gay, Exp. Meth. Phys. Sci. A29, 231 (1995) G Alexander, TAUP-2778-04

[7] A E Bondar and E L Saldin, Nucl. Instrum. Methods 195, 577 (1982)

A E Bondar et al, Proc. 12th Int. Conf. on High Energy Accelerators, 1988, pp. 240243

S A Belomestnykh et al, Nucl. Instrum. Methods A227, 173 (1984)

[8] H R Band et al, Nucl. Instrum. Methods A400, 24 (1997)

P Steiner et al, Nucl. Instrum. Methods A419, 105 (1998)

P S Cooper et al, Phys. Rev. Lett. 34, 1589 (1975)

M V Dyug et al, Nucl. Instrum. Methods A536, 338 (2005)

A V Grigoriev et al, EPAC-2004-THPLT106

[9] G Alexander et al, Nonlin. Phenom. Complex Syst. 8, 180 (2005)

G Alexander and E Reinherz-Aronis, arXiv:hep-ex/0505001

G Alexander, TAUP-2778-04

G Alexander and I Cohen, Nucl. Instrum. Methods A486, 552 (2002)

[10] GEANT4 Collaboration: S Agostinelli et al, Nucl. Instrum. Methods A506, 250 (2003)

[11] R Dollan, K Laihem and A Schälicke, Nucl. Instrum. Methods A559, 185 (2006), arXiv:physics/0512192 